\title{
Ação crônica do napsilato de propoxifeno na prenhez da rata
}

\author{
Chronic effects of propoxyphene napsylate on pregnant rats
}

Eliane T. R. Mendes, Manuel J. Simões, Joaquim Evêncio-Neto, Silvia Espiridião, Luiz Kulay Jr.

\section{RESUM0}

O objetivo deste trabalho é avaliar os efeitos do napsilato de propoxifeno sobre a prenhez da rata albina. Para tanto utilizamos 50 ratas prenhes divididas ao acaso em cinco grupos iguais. Todas receberam diariamente, por gavagem, o volume de $1 \mathrm{ml}$, desde os dias $O$ (zero) até o 20 de prenhez, com as seguintes características: grupo I - somente água destilada (controle); grupo II - solução aquosa de acácia 2\% (veículo); grupos III, IV e V - respectivamente, 5,15 e $45 \mathrm{mg} / \mathrm{kg}$ de peso de napsilato de propoxifeno dissolvido em solução de acácia a $2 \%$. Os pesos maternos foram anotados nos dias 0 (zero), 7, 14 e 20 de prenhez; no $20^{\circ}$ dia as matrizes foram sacrificadas. Nossos resultados mostraram que os animais tratados com 45 $\mathrm{mg} / \mathrm{kg}$ do fármaco apresentaram redução dos pesos individuais dos fetos como também dos pesos das ninhadas e das placentas. Quanto às outras variáveis apreciadas: número de reabsorções, de implantações e de placentas não houve diferença significante entre os grupos tratados em relação ao grupo controle.

PALAVRAS-CHAVE: Propoxifeno. Drogas na gestação. Analgésicos. Placenta.

\section{Introdução}

Analgésico opióide, o napsilato de propoxifeno é consumido pela população, inclusive pelas gestantes nos vários períodos de gravidez. Está situado na categoria "C" do Food and Drug Administration (1980) ${ }^{6}$ : "estudos não controlados ou não conclusivos em animais e humanos: o fármaco pode ser administrado se o benefício for maior do que o potencial de risco".

Como todos os opióides, o napsilato de propoxifeno ultrapassa a placenta tanto no humano ${ }^{15}$ quanto na $\mathrm{rata}^{2}$. Na espécie humana, entre

Departamento de Saúde Materno - Infantil da Faculdade de Medicina do ABC, São Paulo;

Departamento de Tocoginecologia da Universidade Federal de São Paulo / Escola Paulista de Medicina - São Paulo.

Correspondência:

Prof. Dr. Luiz Kulay Júnior

Rua Napoleão de Barros, 715; $8^{\circ}$ Andar.

Hospital São Paulo, Disciplina de Obstetrícia.

CEP 04024-002, São Paulo, Brasil. os conceptos expostos durante o primeiro trimestre, a literatura refere a ocorrência de artrogripose, retardo mental e de crescimento $^{1}$, deformidades múltiplas de extremidades, onfocele e hérnia diafragmática ${ }^{11}$, além de malformações faciais e anoftalmia bilateral ${ }^{7}$. No entanto, 686 conceptos observados por Heinonen e cols. $(1977)^{9}$ e, posteriormente, outros 1029 acompanhados por Rosa $(1993)^{12}$, expostos igualmente durante o período embriogênico, não demonstraram a correlação entre o propoxifeno e defeitos congênitos.

No campo da experimentação, Emmerson e cols. $(1971 \mathrm{a}, \mathrm{b})^{4,5}$, administrando doses diárias de 200-400 mg $/ \mathrm{kg}$ de peso do $6^{\circ}$ ao $15^{\circ}$ dia de prenhez, observaram toxicidade em $20 \%$ das ratas com redução da viabilidade da prole.

Assim, propusemo-nos ministrar doses diferentes de napsilato de propoxifeno, por dia via oral, durante toda a prenhez de ratas, para avaliar a ação sobre a matriz e seus conceptos. 


\section{Material e métodos}

\section{Animais e tratamento}

Utilizamos ratas (EPM-1 Wistar), adultas, virgens, pesando de 150 a $200 \mathrm{~g}$, com regime de alimentação (ração Purina - Labina) e de água à vontade. Foram inicialmente acasaladas na proporção de duas fêmeas para cada macho por um período de duas horas. O teste para prenhez foi o determinado por Hamilton e Wolfe (1938) ${ }^{8}$, ou seja, o encontro de espermatozóides na luz vaginal. As 50 ratas foram então divididas ao acaso em cinco grupos, a saber: I, animais que receberam água (controle); II, animais que receberam solução de acácia a $2 \%$ (veículo); III, animais tratados com 5 $\mathrm{mg} / \mathrm{kg}$ de napsilato de propoxifeno (cedido pelo Laboratório Eli Lilly S.A.) em solução de acácia a $2 \%$; IV, animais tratados com $15 \mathrm{mg} / \mathrm{kg}$ de napsilato de propoxifeno em solução de acácia a $2 \%$ e V, animais tratados com $45 \mathrm{mg} / \mathrm{kg}$ de napsilato de propoxifeno em solução de acácia a $2 \%$. O tratamento teve início logo após a feitura do teste de prenhez (dia 0) e se estendeu até o $20^{\circ}$ dia, sendo que todos os animais receberam diariamente $1 \mathrm{ml}$ de solução, por gavagem, independente do grupo apreciado.

Todos os animais foram pesados nos dias zero, $7,14^{\circ}$ e 20 dia de prenhez. O ganho de peso percentual foi calculado de acordo com a seguinte fórmula:

$\Delta \%=$ (peso no dia $X$ - peso inicial) $\times 100 /$ peso inicial onde $X=$ dia 7 ou 14 ou 20 de prenhez.

Ao termo $\left(20^{\circ}\right.$ dia), todos os animais foram sacrificados sob anestesia profunda com éter etílico; a seguir foi aberta a cavidade abdominal e anotados os seguintes parâmetros: número de sítios de implantação e de reabsorção, número de placentas e de fetos. Foram também determinados os pesos das placentas e dos fetos.

\section{Análise estatística}

Foram realizadas análises paramétricas e não-paramétricas; o nível de rejeição para hipótese de nulidade foi fixada em 5\%. Múltiplas comparações foram feitas pelo teste de KruskalWallis e análise de variância ${ }^{13,14}$.

\section{Resultados}

No que diz respeito ao ganho de peso materno, durante a gestação, nossos resultados não revelaram diferenças estatisticamente significantes entre os vários grupos tratados com diferentes doses de napsilato de propoxifeno (Figura 1).

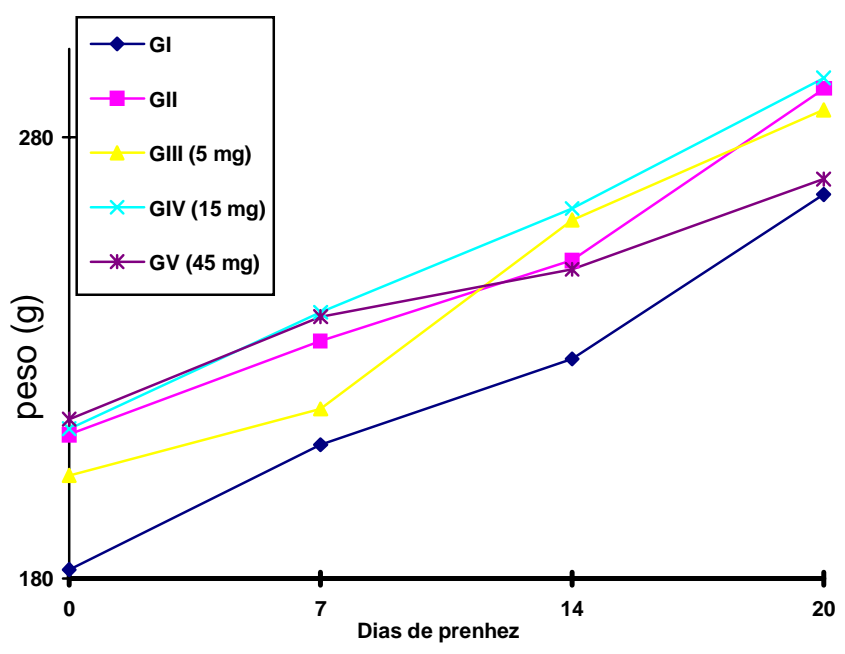

Figura 1 - Evolução do peso $(\mathrm{g})$ das ratas prenhes tratadas com várias dosagens de napsilato de propoxifeno.

Com relação às ratas tratadas com $15 \mathrm{mg} / \mathrm{kg}$ de napsilato de propoxifeno durante toda a prenhez, notamos haver redução estatisticamente significante dos pesos individuais médios dos fetos assim como do peso médio das ninhadas. Já nos animais que receberam $45 \mathrm{mg}$ do fármaco por $\mathrm{kg}$ de peso, além dos efeitos acima referidos, constatamos também diminuição do peso do conjunto de placentas (Tabela 1).

\section{Discussão}

Inicialmente é preciso mencionar que a dose terapêutica, na espécie humana, (300 mg), corresponde a $5 \mathrm{mg} / \mathrm{kg}$ de peso ao dia. Com relação ao peso das ratas, calculado a partir do ganho da diferença percentual através da análise de variância por postos de Friedman, não foi significante, considerados os cinco grupos estudados. Em outras palavras, as várias concentrações administradas não alteraram o ganho de peso corporal no decorrer da prenhez. Nossos resultados, assim, estão em desacordo com as observações de Emmerson e cols. $(1971)^{4}$ que encontraram diminuição de ganho de peso corporal em ratas não prenhes a partir da $2^{\mathrm{a}}$ semana de tratamento, com o uso de 200 a 400 mg de napsilato de propoxifeno misturado à ração alimentar diária, sem alteração de ingesta alimentar.

Quanto ao número de implantações e de reabsorções, nossos resultados não mostraram diferenças significantes. Analisando e comparando 
Tabela 1 - Efeitos do napsilato de propoxifeno sobre os vários parâmetros estudados na rata prenhe.

\begin{tabular}{|c|c|c|c|c|c|}
\hline & GI & GII & GIII (5 mg/kg) & GIV (15 mg/kg) & GV (45 mg/kg) \\
\hline Implantações & $9,70 \pm 2,11$ & $9,68 \pm 2,50$ & $9,10 \pm 0,88$ & $8,50 \pm 3,57$ & $7,14 \pm 3,34$ \\
\hline Reabsorções & $1,0 \pm 0,52$ & $1,50 \pm 3,03$ & $1,0 \pm 0,42$ & $1,17 \pm 0,67$ & $1,20 \pm 3,02$ \\
\hline Número de fetos & $9,50 \pm 2,12$ & $9,11 \pm 2,52$ & $08,70 \pm 0,95$ & $7,80 \pm 3,43$ & $6,57 \pm 2,76$ \\
\hline Peso médio da ninhada (g) & $23,80 \pm 5,96$ & $20,66 \pm 4,58$ & $25,44 \pm 4,72$ & $15,84 \pm 7,14 *$ & $11,88 \pm 7,58 * *$ \\
\hline $\begin{array}{l}\text { Peso individual médio dos } \\
\text { fetos }(\mathrm{g})\end{array}$ & $2,50 \pm 0,30$ & $2,30 \pm 0,24$ & $2,49 \pm 0,26$ & $2,08 \pm 0,32 *$ & $1,68 \pm 0,53 * *$ \\
\hline Número de placentas & $9,50 \pm 2,12$ & $9,11 \pm 2,52$ & $8,70 \pm 0,95$ & $7,80 \pm 3,41$ & $6,57 \pm 2,76$ \\
\hline $\begin{array}{l}\text { Peso do conjunto das } \\
\text { placentas }(\mathrm{g})\end{array}$ & $4,74 \pm 1,16$ & $4,20 \pm 1,11$ & $4,54 \pm 0,76$ & $3,33 \pm 1,57$ & $3,06 \pm 1,21^{*}$ \\
\hline $\begin{array}{l}\text { Peso individual médio das } \\
\text { placentas }(\mathrm{g})\end{array}$ & $0,50 \pm 0,04$ & $0,47 \pm 0,04$ & $0,52 \pm 0,03$ & $0,43 \pm 0,05$ & $0,48 \pm 0,05$ \\
\hline
\end{tabular}

* Significantemente diferente dos outros grupos $(p<0,05)$

** Significantemente diferente dos outros grupos $(\mathrm{p}<0,05)$

o número de reabsorções com o número de implantações de placentas e de fetos, entre os vários grupos, percebemos que o napsilato de propoxifeno não interferiu no processo da implantação. Nossos resultados, assim, estão de acordo com os achados de Emmerson e cols. $(1971)^{5}$ que, embora utilizando doses maiores, não encontraram alterações no número de implantações de blastocistos no seu experimento.

No que diz respeito ao conjunto das placentas, notamos que seu peso no GV foi o menor de todos com redução de aproximadamente $36 \%$ quando comparado com o GI. Guanto aos demais grupos, a análise estatística não mostrou diferenças significantes. A redução do peso no grupo $\mathrm{V}$, tratado com $45 \mathrm{mg} / \mathrm{kg}$, dose nove vezes maior do que a dose terapêutica para o ser humano, demonstra que, de certa maneira, a droga por nós utilizada foi lesiva. No entanto, quando comparamos o peso individual médio das placentas, nos vários grupos estudados, verificamos não haver diferença estatisticamente significante. Convém lembrar que o peso da placenta humana corresponde em média a 1/6 do peso fetal, no termo da gravidez. Em animais de múltiplas crias, também a relação ponderal feto/placenta é sempre proporcional. Assim, acreditamos que o número reduzido de fetos no grupo $\mathrm{V}$, embora não significante, pode ter influído no peso do conjunto das placentas.

Com relação ao peso dos conceptos, foi observada redução, tanto do peso corporal individual, quanto das ninhadas, nos grupos que receberam 15 e $45 \mathrm{mg} / \mathrm{kg}$. Esses resultados indicam que a droga, de certo modo, atuou sobre a homeostase fetal, alterando seu peso. O desenvolvimento fetal, sabemos, obedece a três fases: hiperplasia, hipertrofia e maturação, na qual ocorre o desenvolvimento dos órgãos e a respectiva diferenciação celular. Emmerson e cols. (1971) ${ }^{5}$, ministrando o fármaco até o $15^{\circ} \mathrm{dia}$, fase inicial de crescimento correspondente aos períodos de hipertrofia e hiperplasia, nada observaram quanto à alteração de peso dos conceptos.

Nosso experimento, porém, mostrou crias com baixo peso no $20^{\circ}$ dia de prenhez. Esse resultado, nos parece estar associado a dois fatos. Primeiro ao fato de que no $18^{\circ}$ dia de prenhez da rata inicia-se um processo fisiológico de reepitelização do útero, 
determinando redução gradual e progressivo da área de inserção placentária ${ }^{3,10} \mathrm{e}$, segundo, à interferência do fármaco no ganho de peso das ratas ${ }^{4}$. Embora não tenhamos observado diminuição do peso das ratas no termo das gestações acreditamos que no presente estudo o fármaco interferiu diretamente sobre os conceptos, diminuindo o aporte de elementos plásticos, essenciais para o desenvolvimento fetal. Esses dados, a nosso ver, podem, assim, justificar o baixo peso das crias expostas a doses de 15 e $45 \mathrm{mg} / \mathrm{kg}$ de napsilato de propoxifeno, respectivamente 3 e 9 vezes maior do que a dose terapêtica para humanos, durante todo o processo de prenhez. É importante ressaltar ainda, que a redução de peso por nós observada foi diretamente proporcional às doses empregadas.

Finalmente, podemos dizer, também, que por mais lesiva que seja sua ação o fármaco não desenvolveu potencial necessário para produzir malformações, seja em doses altas ${ }^{4,5}$ ou em baixas doses com as quais trabalhamos.

\section{SUMMARY}

The purpose of this study is to evaluate the effects of propoxyphene napsylate on the pregnancy of the rat. We used fifty pregnant rats divided into five groups. All the animals received daily $1 \mathrm{ml}$ of the solution by gavage from day 0 to the 20th day of pregnancy. Group I - only distilled water (control); group II - aqueous solution of acacia $2 \%$ (vehicle); groups III, IV and V-respectively, 5, 15 and $45 \mathrm{mg} / \mathrm{kg}$ of weight of propoxyphene napsylate diluted in $2 \%$ acacia solution. The animals were weighed on days $0,7,14$ and 20 of pregnancy. All animals were sacrificed on the 20th day of pregnancy. Our results showed that the animals treated with $45 \mathrm{mg} / \mathrm{kg}$ of propoxyphene napsylate presented reduction of the individual weights of the fetuses, as well as of the weights of the newborns and placentas. The difference betewwn number of resorptions, implantations and placentas of the treated groups was shown to be non significant cohen compered with the control groups.

KEY WORDS: Propoxyphene napsylate. Placenta. Analgesic. drugs in pregnancy.

\section{Referências bibliográficas}

1. Barrow MV, Souder DE. Propoxyphene and congenital malformations. JAMA 1971; 217: 1551-2.

2. Due SL, Sullivan HR, Cochrane RL, Page JG, Mc Mahon RE. Maternal and fetal blood brain distribution of d-propoxyphene and nor propoxyphene in rat and dog. Toxicol Appl Pharmacol 1981; 58: 194-202.

3. Duval M. Le placenta des rongeurs. III Le placenta de la souris et du rat. J Anat 1891; 27: 515-612.

4. Emmerson JL, Gibson WR, Anderson RC. Acute toxicity of propoxyphene salts. Toxicol Appl Pharmacol 1971; 19: 445-51.

5. Emmerson JL, Owen NV, Koenig GR, Markhan JK, Anderson RC. Reproduction and teratology studies on propoxyphene napsylate. Toxicol Appl Pharmacol 1971; 19: 471-9.

6. Food and Drug Administration. Federal Register 1980, 44: 37433-4.

7. Golden SM, Perman KL. Bilateral clinical anophtalmia: drugs as potential factors. South Med J 1980; 73: $1404-7$.

8. Hamilton JB, Wolfe JM. The effect of male hormone substance upon birth and prenatal development in the rat. Anat Rec 1938; 70: 433-40.

9. Heinonen OP, Slone D, Shapiro S. Birth defects and drugs in pregnancy. Littleton, M.A Publishing Sciences Group; 1977.

10. Kulay L, Jr. Histochemical study (polysaccharides, lipids and proteins) of rat's placenta, during the functional period. Acta Anat 1968; 70: 607-22.

11. Ringrose CAD. The hazard of neurotropic drugs in the fertile years. Can Med Assoc J 1972; 106: 1058.

12. Rosa F. Personal communication. Federal Register 1993; 57: 3643-5.

13. Siegel S. Estatística no parametrica - México: Trilla; 1975. 346p.

14. Sokal RR, Rohlf FJ. Biometry. San Francisco: W.H. Freeman; 1969. 776p.

15. Tyson HK. Neonatal withdrawal symptoms associated with maternal use of propoxyphene hydrochloridre (Darvon). J Pediatr 1974; 85: 684-5. 